\title{
L'arbitrage entre investisseur et État face aux derniers accords commerciaux régionaux
}

\author{
By \\ DR. Charles-Emmanuel
}

Côté est professeur titulaire à la Faculté de droit de

l'Université Laval 


\section{Notice biographique}

Charles-Emmanuel Côté est professeur titulaire à la Faculté de droit de l'Université Laval, où il enseigne le droit international public, le droit international économique et le droit constitutionnel. Il est codirecteur du Centre de droit international et transnational (CDIT) et membre du Centre d'études pluridisciplinaires sur le commerce et les investissements internationaux (CEPCI) de l'École supérieure d'études internationales (ÉSÉI). Il a été Vice-doyen aux études de premier cycle et à la formation continue et Secrétaire de la Faculté de droit de 2014 à 2018. Avocat au Barreau du Québec, il est Senior Fellow au Centre for International Governance Innovation (CIGI) et membre du Conseil d'administration du Conseil canadien de droit international (CCDI). Le professeur Côté a auparavant été conseiller en politiques institutionnelles et constitutionnelles au Secrétariat aux affaires intergouvernementales canadiennes (SAIC) du gouvernement du Québec, ainsi que chercheur au Centre de droit de la consommation de l'Université catholique de Louvain, en Belgique, œuvrant sur des projets d'assistance législative de la Commission européenne à destination des pays d'Europe centrale et orientale et de l'ex-URSS. Il est membre du Committee on the Rule of Law and International Investment Law de la International Law Association (ILA) et a été nommé sur la liste des personnes pouvant agir à titre de membre des groupes spéciaux de l'Accord de libre-échange canadien 
(ALEC) et du chapitre 19 de l'Accord de libre-échange nordaméricain (ALENA). Son ouvrage intitulé La participation des personnes privées au règlement des différends internationaux économiques : l'élargissement du droit de porter plainte à l'OMC s'est mérité un prix de la Chambre de commerce internationale.

\section{Résumé}

L'arbitrage entre investisseur et État fondé sur un accord international permet aux entreprises étrangères de présenter directement une réclamation contre l'État où elles ont investi, afin d'obtenir réparation du préjudice causé par une mesure contraire à cet accord. En raison du consentement préalable donné par les États à cet arbitrage, ainsi que de la portée très large de la protection offerte aux entreprises étrangères par ces accords sur l'investissement, l'arbitrage entre investisseur et État suscite une grande controverse tant dans les pays industrialisés que dans les pays en développement. Le présent article analyse comment cette controverse s'est répercutée de manière fort différente dans les chapitres sur l'investissement de trois accords commerciaux régionaux conclus récemment, soit le Partenariat transpacifique global et progressiste (PTPGP), l'Accord économique et commercial global entre l'Union européenne et le Canada (AECG) et l'Accord entre le Canada, les États-Unis et le Mexique $(A C E U M)$. Trois modèles se distinguent: celui du maintien de l'arbitrage entre investisseur et État, celui de sa transformation et 
enfin celui de son abandon. En guise de conclusion, quelques recommandations sont formulées à l'intention du gouvernement égyptien à la lumière de ces développements récents.

\begin{abstract}
Investor-state arbitration based on an international agreement allows foreign companies to directly file a claim against the state where they have invested, in order to obtain compensation for the damage caused by a measure contrary to that agreement. Because of the prior consent given by the states to this arbitration, as well as the very broad scope of the protection offered to foreign companies by these investment agreements, the arbitration between investor and state arouses great controversy in both industrialized and developing countries. This article analyses how this controversy has had a very different impact on the investment chapters of three recently concluded regional trade agreements, the Comprehensive and Progressive Trans-Pacific Partnership (CPTPP), the Comprehensive Economic and Trade Agreement between the European Union and Canada (CETA) and the Agreement between Canada, the United States and Mexico (CUSMA). Three models stand out : that of maintaining arbitration between investor and State, that of its transformation and finally that of its abandonment. In conclusion, some recommendations are made to the Egyptian government in light of these recent developments.
\end{abstract}




\section{Introduction}

Différents modèles coexistent actuellement dans les plus récents accords commerciaux régionaux en matière de règlement des différends entre investisseur étranger et État (RDIE). En effet, trois approches très différentes se dégagent de la pratique conventionnelle récente des États avec l'Accord économique et commercial global entre le Canada et l'Union européenne $e^{(1)}$ (AECG) signé en 2016, le Partenariat transpacifique global et progressiste $^{(2)}$ (PTPGP) signé en 2018, ainsi que l'Accord entre le Canada, les États-Unis et le Mexique ${ }^{(3)}$ (ACEUM) également signé en 2018.

(1) Accord économique et commercial global entre le Canada, d'une part, et l'Union européenne et ses États membres, d'autre part, 30 octobre 2016, en ligne: $\quad$ http://www.international.gc.ca/trade-commerce/trade-agreementsaccords-commerciaux/agr-acc/ceta-aecg/text-texte/toc-tdm.aspx?lang=fra [AECG].

(2) Partenariat transpacifique global et progressiste, 8 mars 2018, en ligne: http://international.gc.ca/trade-commerce/trade-agreements-accordscommerciaux/agr-acc/cptpp-ptpgp/text-texte/cptpp-ptpgp.aspx?lang=fra (Australie, Brunei Darussalam, Canada, Chili, Japon, Malaisie, Mexique, Nouvelle-Zélande, Pérou, Singapour et Viêt-Nam) [PTPGP].

(3) Accord entre le Canada, les États-Unis et le Mexique, 30 novembre 2018, en ligne: https://www.international.gc.ca/trade-commerce/trade-agreementsaccords-commerciaux/agr-acc/cusma-aceum/text-texte/toc-tdm.aspx?lang=fra [ACEUM]. 
Il convient de rappeler que l'arbitrage entre investisseur étranger et État est né graduellement de la volonté des États d'attirer des capitaux étrangers, en affranchissant leur protection juridique de la seule souveraineté de l'État d'accueil. ${ }^{(1)}$ Cet affranchissement a été réalisé par la délocalisation de cette protection au moyen de règles matérielles fixées par des traités bilatéraux d'investissement (TBI), ainsi qu'en confiant le règlement des différends entre investisseur et État à des tribunaux arbitraux plutôt qu'aux juridictions de l'État d'accueil. En évitant d'avoir à recourir à la protection diplomatique de l'État d'origine de l'investisseur, l'arbitrage a aussi permis la dépolitisation du règlement de ces différends, en les confinant aux rapports entre les parties directement concernées ${ }^{(2)}$. La Convention de Washington de 1965, ou Convention pour le règlement des différends relatifs aux investissements entre États et ressortissants d'autres États $^{(3)}$ (Convention CIRDI), qui a institué le Centre

(1) Voir généralement Charles Leben, «Droit international des investissements : un survol historique », dans Charles Leben, dir, Droit international des investissements et de l'arbitrage transnational, Paris, Pedone, 2015, 1; Arnaud de Nanteuil, Droit international de l'investissement, Paris, Pedone, 2014 aux pp 11-51.

(2) Ibrahim F.I. Shihata, «Towards a Greater Depoliticization of Investment Disputes : The Roles of ICSID and MIGA» (1986) 1 ICSID Review 47.

(3) Convention pour le règlement des différends relatifs aux investissements entre États et ressortissants d'autres États, 18 mars 1965, 575 RTNU 160. Voir généralement Christoph Schreuer, The ICSID Convention: A Commentary, $2^{\mathrm{e}}$ éd, Cambridge, Cambridge University Press, 2009. 
international pour le règlement des différends relatifs à l'investissement (CIRDI), constitue un jalon important de la construction de ce système, avec la création d'un arbitrage entièrement délocalisé et rattaché exclusivement au droit international public. Enfin le système a été parachevé avec la reconnaissance par les tribunaux arbitraux de la validité du consentement dissocié à l'arbitrage, dans les affaires Southern Pacific Properties (Middle East) c Égypte ${ }^{(1)}$ de 1988 et Asian Agricultural Products Limited c Sri Lanka ${ }^{(2)}$ de 1990. Le consentement des parties à l'arbitrage est réalisé par la combinaison de l'offre unilatérale d'arbitrage faite par l'État dans la clause d'arbitrage du traité avec l'exercice postérieur d'un recours par l'investisseur ${ }^{(3)}$. L'arbitrage entre investisseur et État permet ainsi à l'investisseur qui s'estime lésé de réclamer directement des dommages-intérêts auprès d'un tribunal international pour violation des règles matérielles du traité par une mesure de l'État. Il faut noter que de nombreux accords commerciaux régionaux

(1) Southern Pacific Properties (Middle East) c Égypte, Sentence sur la compétence, Aff CIRDI n ARB/84/3 (Loi égyptienne, 14 avril 1988).

(2) Asian Agricultural Products Limited c Sri Lanka, Sentence finale, Aff CIRDI $\mathrm{n}^{\circ}$ ARB/87/3 (TBI Sri Lanka-Royaume-Uni, 27 juin 1990).

(3) Voir généralement Jan Paulsson, «Arbitration without Privity » (1995) 10 ICSID Review 232. 
comportent aussi un chapitre sur l'investissement dont le contenu est similaire à un $\mathrm{TBI}^{(1)}$.

L'arbitrage entre investisseur et État a pris son véritable envol relativement récemment, à la fin des années 1990, notamment avec l'affaire Ethyl Corporation c Canada ${ }^{(2)}$ de 1998, où une mesure environnementale canadienne a fait l'objet d'une réclamation de la part d'une entreprise privée américaine. La controverse n'a pas tardé à éclater au sujet de ce type d'arbitrage, à la fois dans les pays industrialisés et dans les pays en développement ${ }^{(3)}$. La légitimité même du droit d'action des investisseurs étrangers est remise en question alors qu'il peut s'exercer sans épuisement des recours internes et sans filtre politique. D'aucuns craignent que les

(1) La Conférence des Nations Unies sur le commerce et le développement (CNUCED) tient une base de données qui recense tous ces accords internationaux sur son portail sur l'investissement. Investment Policy Hub, en ligne : CNUCED https://investmentpolicy.unctad.org/.

(2) Ethyl Corporation c Canada, Sentence sur la compétence, CNUDCI (ALENA, 24 juin 1998).

(3) Voir généralement Roberto Echandi, «The Debate on Investor-State TreatyBased Dispute Settlement: Empirical Evidence (1987-2017) and Policy Implications » (2019) 39 ICSID Review 32; Charles-Emmanuel Côté, La participation des personnes privées au règlement des différends internationaux économiques : l'élargissement du droit de porter plainte à l'OMC, Bruxelles, Bruylant, 2007 aux pp 265-308; Susan D. Franck, « The Legitimacy Crisis in Investment Treaty Arbitration: Privatizing Public International Law Through Inconsistent Decisions » (2005) 73 Fordham Law Review 1521. 
gouvernements s'abstiennent de légiférer dans les domaines de la protection de la santé ou de l'environnement, de peur de faire face à une réclamation, ce qui créerait un «regulatory chill» ou gel réglementaire. ${ }^{(1)}$ La portée incertaine de la protection matérielle des investissements étrangers inquiète aussi, avec des normes à caractère très général comme la clause du traitement juste et équitable (TJE), la clause de la nation la plus favorisée (NPF), la notion d'expropriation indirecte ou encore la clause de couverture des engagements ou «umbrella clause». Le caractère « déjuridictionnalisé » de l'arbitrage entre investisseur et État est aussi problématique, avec de nombreuses divergences entre sentences arbitrales rendues par des tribunaux ad hoc, l'absence de révision sur le fond des sentences, le caractère secret des règlements amiables ou encore de potentiels conflits d'intérêts réels ou apparents entre arbitres et conseillers juridiques. La désarticulation entre le contrôle de la légalité des sentences par les tribunaux du lieu du for et celui de leur exécution forcée par les tribunaux du lieu où se trouvent les actifs de l'État pose aussi des

(1) Voir Donald M. McRae, «Trade and Environment: Competition, Cooperation or Confusion? » (2003) 41 Alberta Law Review 745 à la p 759; Lucien J. Dooghe, «The Revenge of the Trail Smelter: Environmental Regulation as Expropriation Pursuant the North American Free Trade Agreement » (2001) 38 American Business Law Journal 475 aux pp 549-550. 
difficultés dans l'arbitrage hors CIRDI. ${ }^{(1)}$ Face à cette controverse, certains États comme la Bolivie, l'Équateur et le Venezuela ont choisi de se retirer de la Convention de Washington, voire même de tous ses TBI dans le cas de l'Équateur ${ }^{(2)}$.

Il convient d'analyser comment cette controverse s'est répercutée de manière fort différente dans les chapitres sur l'investissement du PTPGP, de l'AECG et de l'ACEUM, trois accords commerciaux régionaux conclus récemment. Trois modèles se distinguent : celui du maintien de l'arbitrage entre investisseur et État (2), celui de sa transformation (3) et enfin celui de son abandon (4). En guise de conclusion, quelques recommandations sont formulées à l'intention du gouvernement égyptien à la lumière de ces développements récents (5).

(1) Dans l'arbitrage CIRDI, le Comité ad hoc peut suspendre la sentence pendant l'examen d'une demande d'annulation. Convention CIRDI, supra note 3, art $52(5)$.

(2) Leon E. Trakman, «The ICSID Under Siege» (2012) 45 Cornell International Law Journal 603 à la p 604; International Institute for Sustainable Development, «L'Équateur dénonce ses 16 TBI restants et publie le rapport d'audit de la CAITISA », Investment Treaty News (12 juin 2017), en ligne: IISD https://www.iisd.org/itn/fr/2017/06/12/ecuador-denounces-itsremaining-16-bits-and-publishes-caitisa-audit-report/. 


\section{Premier modèle : le maintien de l'arbitrage entre investisseur et État dans le PTPGP}

Le $P T P G P$ a été signé par 11 pays de la zone Pacifique, le 8 mars 2018, et est entré en vigueur le 30 décembre 2018 entre six premiers pays. Cet accord incorpore presqu'intégralement le défunt Partenariat transpacifique ${ }^{(1)}(P T P)$ signé en 2016 et abandonné par les États-Unis l'année suivante après l'élection du Président Trump. Le PTPGP reprend le modèle dominant de l'arbitrage entre investisseur et État présent dans la plupart des TBI et le diffuse dans la zone Pacifique, y compris entre des pays industrialisés comme l'Australie, le Canada et le Japon. Son chapitre 9 sur l'investissement comporte assez peu d'innovations juridiques et reprend essentiellement l'approche traditionnelle des États-Unis en la matière ${ }^{(2)}$. Les règles de fond qu'il prescrit visent à la fois la

(1) Partenariat transpacifique, 4 février 2016, en ligne : http://international.gc.ca/trade-commerce/trade-agreements-accordscommerciaux/agr-acc/tpp-ptp/text-texte/toc-tdm.aspx?lang=fra (Australie, Brunei Darussalam, Canada, Chili, États-Unis, Japon, Malaisie, Mexique, Nouvelle-Zélande, Pérou, Singapour et Viêt-Nam) $[P T P]$. Par souci de clarté, la référence aux articles du PTP incorporés au PTPGP est indiquée comme une référence au $P T P G P$.

(2) Une étude a démontré que $81 \%$ du texte du chapitre sur l'investissement du PTP correspond à la pratique conventionnelle antérieure des États-Unis. Wolfgang Alschner et Dmitriy Skougarevsiy, «The New Gold Standard? Empirically Situating the Trans-Pacific Partnership in the Investment Treaty Universe » (2016) 17 Journal of World Investment \& Trade 339 at 353. Voir

$=$ 
phase pré-investissement et la phase post-investissement ${ }^{(1)}$, la clause du TJE est limitée à la norme minimale de traitement des étrangers du droit coutumier international ${ }^{(2)}$, tandis que la clause d'expropriation est assortie d'une annexe interprétative visant à circonscrire la notion d'expropriation indirecte ${ }^{(3)}$. Une clause sur le droit de l'État de légiférer est tout de même ajoutée ${ }^{(4)}$, de même que certaines précisions importantes sur la portée de la clause NPF et de la clause du traitement national ${ }^{(5)}$.

Le modèle traditionnel de l'arbitrage entre investisseur et État est repris, avec l'ajout d'une clause de transparence prévoyant la publicité des audiences et des documents de procédure, ainsi que la reconnaissance du pouvoir des arbitres de recevoir des mémoires de

généralement José Alvarez, «Is the Trans-Pacific Partnership's Investment Chapter the New "Gold Standard"?» (2016) 47 Victoria University of Wellington Law Review 503.

(1) PTPGP, supra note 2, art 9.1, sub verbo « investisseur d'une Partie », 9.4 et 9.5.

(2) Ibid art 9.6.

(3) Ibid art 9.8 et annexe 9-B.

(4) Ibid art 9.16.

(5) Ibid art 9.4 et 9.5. Par exemple, les objectifs légitimes de bien-être public visés par une distinction doivent être pris en considération dans l'établissement de la similarité des circonstances dans lesquelles se trouvent des investisseurs ou leurs investissements (ibid art 9.4 note 14). 
tierces parties ou «amicus curiae $»^{(1)}$. Le défunt PTP prévoyait à l'instar des TBI américains deux autres causes d'action, en plus de la violation des règles matérielles du traité, soit la violation du contrat d'État ou celle de l'autorisation administrative d'investissement ${ }^{(2)}$. Ces causes d'action additionnelles ont été abandonnées lors de l'incorporation du PTP dans le PTPGP $P^{(3)}$.

Bien qu'il maintienne pour l'essentiel le modèle traditionnel de l'arbitrage entre investisseur et État, certaines particularités du PTPGP doivent néanmoins être soulignées. D'une part une clause originale permet à un État d'exclure, s'il le désire, les réclamations visant les politiques de lutte contre le tabagisme, dans la foulée de la réclamation infructueuse de la société multinationale Philip Morris contre la politique australienne sur l'emballage neutre des produits du tabac dans l'affaire Philip Morris Asia c Australie ${ }^{(4)} \mathrm{de}$ $2015^{(5)}$. D'autre part, certains pays ont éliminé l'arbitrage entre investisseur et État dans leurs rapports mutuels, ou encore l'ont

(1) Ibid art 9.23 et 9.24 .

(2) Ibid art 9.19.1 a) i) (B) et (C) et 9.19 .1 b) i) (B) et C).

(3) Ibid art 2, annexe, paragr 2(b).

(4) Philip Morris Asia c Australie, «Sentence sur la compétence et la recevabilité », Aff CPA n 2012-12 (TBI Australie-Hong Kong, 17 décembre 2015).

(5) PTPGP, supra note 2, art 29.5. 
assujetti à des conditions additionnelles, au moyen d'une série d'échanges de lettres. Ainsi, l'Australie et la Nouvelle-Zélande refusent ce système de règlement des différends entre elles ${ }^{(1)}$, tandis que la Nouvelle-Zélande le refuse aussi dans ses rapports avec le Pérou $^{(2)}$, alors qu'elle refuse la dissociation du consentement et rétablit l'exigence du compromis d'arbitrage au cas par cas avec le Brunei, la Malaisie et le Vietnam ${ }^{(3)}$. Enfin, quatre pays introduisent une clause «fork-in-the-road», par laquelle le choix par l'investisseur de soumettre sa réclamation aux juridictions nationales est définitif et ferme l'accès à l'arbitrage (le Chili, le

(1) Échange de lettres entre la Nouvelle-Zélande et l'Australie concernant le règlement des différends entre investisseur et État, 8 mars 2018, en ligne: https://www.mfat.govt.nz/assets/CPTPP/New-Zealand-Australia-ISDS-TradeRemedies-and-Relationship-with-Other-Agreements.pdf.

(2) Échange de lettres entre la Nouvelle-Zélande et le Pérou concernant le règlement des différends entre investisseur et État, 8 mars 2018, en ligne: https://www.mfat.govt.nz/assets/CPTPP/New-Zealand-Peru-ISDS.pdf.

(3) Échange de lettres entre la Nouvelle-Zélande et le Brunéi Darussalam concernant le règlement des différends entre investisseur et État, 8 mars 2018, en ligne: https://www.mfat.govt.nz/assets/CPTPP/New-ZealandBrunei-ISDS.pdf; Échange de lettres entre la Nouvelle-Zélande et la Malaisie concernant le règlement des différends entre investisseur et État, 8 mars 2018, en ligne: https://www.mfat.govt.nz/assets/CPTPP/New-ZealandMalaysia-ISDS.pdf; Échange de lettres entre la Nouvelle-Zélande et le Vietnam concernant le règlement des différends entre investisseur et État, 8 mars 2018, en ligne: https://www.mfat.govt.nz/assets/CPTPP/New-ZealandViet-Nam-ISDS.pdf. 
Mexique, le Pérou et le Vietnam) ${ }^{(1)}$. Ainsi, l'arbitrage entre investisseur et État est maintenu par le $P T P G P$, malgré certaines atténuations, offrant une réponse assez décousue aux préoccupations nées de la controverse entourant le système.

\section{Deuxième modèle : la transformation de l'arbitrage entre investisseur et État dans l' $A E C G$}

L'AECG propose une transformation profonde du modèle dominant de l'arbitrage entre investisseur et État, en guise de réponse aux préoccupations concernant ce système. L'accord a été signé le 30 octobre 2016, non sans avoir d'abord traversé une crise en raison du blocage tardif de la signature de l'Union européenne (UE) par la Belgique $^{(2)}$. Les raisons de ce blocage concernaient essentiellement le chapitre 8 sur l'investissement et faisaient écho à la controverse

(1) PTPGP, supra note 2, annexe 9-J. Voir Rudolf Dolzer et Christoph Schreuer, Principles of International Investment Law, 2e éd, Oxford, Oxford University Press, 2012 aux pp 267-268.

(2) Voir Charles-Emmanuel Côté, «Investissement - Chronique de droit international économique en 2016 / Digest of International Economic Law in 2016" (2016) 54 Annuaire canadien de droit international 464 aux pp 474478. 
entourant l'arbitrage entre investisseur et État. L'appui de la Belgique a finalement été obtenu en échange d'une série de conditions, dont le refus de la mise en application provisoire du chapitre sur l'investissement et la demande d'un avis préjudiciel à la Cour de justice de l'UE sur la compatibilité de l'arbitrage entre investisseur et État avec le droit de l'UE et le principe de l'exclusivité de la compétence de cette Cour. Par conséquent, même si l'AECG est appliqué provisoirement depuis le 21 septembre 2017 en attendant sa ratification, l'essentiel de son chapitre sur l'investissement est exclu de cette application provisoire, à commencer par le $\operatorname{RDIE}^{(1)}$.

Le chapitre sur l'investissement de l'AECG comporte plusieurs innovations juridiques tant sur le fond que sur le plan du RDIE. L'application des règles de fond à la phase pré-investissement est restreinte et la clause du TJE n'est pas limitée à la norme minimale de traitement des étrangers du droit coutumier international, mais elle est encadrée au moyen d'une liste exhaustive de mesures

(1) UE, Notification concernant l'application provisoire de l'accord économique et commercial global (AECG) entre le Canada, d'une part, et l'Union européenne et ses États membres, d'autre part, [2017] JOUE n L 238/9. Les dispositions du chapitre sur l'investissement s'appliquent à titre provisoire aux investissements étrangers directs, à l'exclusion notable de la clause du TJE, de la clause d'expropriation, ainsi que de l'ensemble du système juridictionnel de RDIE. 
incompatibles et d'un mécanisme de surveillance ${ }^{(1)}$. La notion d'expropriation indirecte est aussi circonscrite par une annexe interprétative et le droit de légiférer de l'État est affirmé de manière claire, alors que des exceptions générales permettent de justifier les violations à certaines règles de fond ${ }^{(2)}$. Une déclaration interprétative a été adoptée par les parties au moment de la signature de l'AECG, afin de réaffirmer le droit des État de légiférer ainsi que de rappeler que son chapitre sur l'investissement marque un changement radical avec le modèle dominant de l'arbitrage entre investisseur et État ${ }^{(3)}$. La question du statut juridique de cette déclaration se pose, elle qui a été adoptée pour répondre aux préoccupations de la Belgique.

Mais c'est sur le plan du règlement des différends que l'AECG innove le plus. À la demande de l'UE, l'arbitrage traditionnel a été remplacé par la création d'un tribunal permanent, coiffé d'un

(1) AECG, supra note 1, art 8.2.4 et 8.10.

(2) Ibid art 8.9.1, 8.12 et annexe A et 28.3.2.

(3) Instrument interprétatif commun concernant l'accord économique et commercial global (AECG) entre le Canada, d'une part, et l'Union européenne et ses Etats membres, d'autre part, 29 octobre 2016, paragr 6 i), en ligne: https://www.international.gc.ca/trade-commerce/trade-agreementsaccords-commerciaux/agr-acc/ceta-aecg/jii-iic.aspx?lang=fra. 
tribunal d'appel ${ }^{(1)}$. Ce système juridictionnel se veut une réponse originale aux préoccupations nées de la controverse entourant le RDIE, en procédant à sa juridictionnalisation. Les quinze membres $\mathrm{du}$ tribunal permanent doivent être nommés par les États sans le concours des investisseurs, pour un mandat de cinq ans renouvelable une fois ${ }^{(2)}$. Les affaires doivent être instruites en division de trois membres nommés par le Président du tribunal et non par les parties au litige ${ }^{(3)}$. Les membres du tribunal doivent recevoir une rétribution mensuelle afin de garantir leur disponibilité et leur emploi à temps plein est envisagé ${ }^{(4)}$. La juridiction d'appel est aussi envisagée mais l'AECG reporte à une décision ultérieure des parties le soin de préciser les détails de son organisation ${ }^{(5)}$. Ce nouveau système juridictionnel de RDIE est censé être transitoire en attendant la mise sur pied d'un tribunal multilatéral de l'investissement ${ }^{(6)}$. En plus de cette transformation profonde $d u$ système, l'AECG ferme la porte aux réclamations relatives à la

\footnotetext{
(1) AECG, supra note 1, art 8.27 et 8.28.

(2) Ibid art 8.27.2 et 8.27.5.

${ }^{(3)}$ Ibid art 8.27.6 et 8.27.7.

(4) Ibid art 8.27.12.

${ }^{(5)}$ Ibid art 8.28.7.

${ }^{(6)}$ Ibid art 8.29.
} 
phase pré-investissement, de même qu'en cas de corruption ou de conduite frauduleuse de la part de l'investisseur ${ }^{(1)}$.

La création d'un tribunal permanent avec une juridiction d'appel et la nomination de ses membres par les États colle davantage avec la dimension de droit international public des différends entre investisseur et État et permet d'espérer une plus grande cohérence entre les sentences arbitrales. Les risques de conflits d'intérêts sont aussi diminués, de même que la tentation d'allonger indûment les procédures. L'introduction de l'appel dans le RDIE est une innovation majeure puisqu'il permettra le réexamen au fond des décisions de première instance, à l'instar du mécanisme de règlement des différends de l'Organisation mondiale $\mathrm{du}$ commerce $(\mathrm{OMC})^{(2)}$. La création de ce tribunal permanent soulève néanmoins de nombreuses difficultés juridiques, en raison de son intégration dans le système global de l'arbitrage entre investisseur et État ${ }^{(3)}$. En effet, l'ensemble des règles sur le contrôle

${ }^{(1)}$ Ibid art 8.2.4, 8.18.1 et 8.18.3.

(2) Voir Mémorandum d'accord sur les règles et procédures régissant le règlement des différends, annexe 2 de l'Accord de Marrakech instituant l'Organisation mondiale du commerce, 15 avril 1994, 1867 RTNU 3; Dominique Carreau et al, Droit international économique, $6^{\mathrm{e}}$ éd, Paris, Dalloz, 2017 aux pp 119-156; Éric Canal-Forgues, Le règlement des différends à l'OMC, $3^{\mathrm{e}}$ éd, Bruxelles, Bruylant, 2008.

(3) Voir August Reinisch, «Will the EU's Proposal Concerning an Investment Court System for CETA and TTIP Lead to Enforceable Awards?-The Limits $=$ 
de la légalité des sentences par les tribunaux du lieu du for ainsi que la reconnaissance et l'exécution des sentences demeurent applicables aux décisions émanant du tribunal permanent. L'astuce permettant d'insérer une instance d'appel dans ce système consiste à ne pas considérer la sentence comme définitive tant que le délai pour faire appel n'est pas écoulé ou que la décision en appel n'a pas été rendue ${ }^{(1)}$. La question de la compatibilité de cette conception avec les règles d'arbitrage se pose, notamment en ce qui concerne le caractère exécutoire des sentences arbitrales. La législation du lieu du for donnera-t-elle effet à cette conception en cas de demande d'annulation de la décision de première instance? L'interdiction de demander l'annulation ou l'exécution de la sentence arbitrale est-elle suffisante pour empêcher un investisseur de le faire? Enfin la voie de l'arbitrage CIRDI semble fermée à l'UE, puisque la Convention de Washington n'envisage pas la possibilité qu'une des parties soit une organisation internationale ${ }^{(2)}$.

of Modifying the ICSID Convention and the Nature of Investment Arbitration » (2016) 19 Journal of International Economic Law 761; Elsa Sardhinha, « Towards a New Horizon in Investor-State Dispute Settlement? Reflections on the Investment Tribunal System in the Comprehensive Economic Trade Agreement (CETA)» (2016) 54 Annuaire canadien de droit international 311.

(1) AECG, supra note 1 art 8.28.9 c), d) et e).

(2) Convention CIRDI, supra note 3, art 67. 
La ratification de l'AECG est semée d'embuches en raison de la controverse entourant son chapitre sur l'investissement et de la question de sa compatibilité avec le droit de l'UE. Dans son arrêt Slowakische Republik c Achmea ${ }^{(1)}$ de 2018, la Cour de justice de l'UE a jugé que les TBI entre États membres sont incompatibles avec le droit de l'UE. En revanche, la Cour de justice a conclu en 2019, dans l'affaire du renvoi préjudiciel de la Belgique, que le chapitre sur l'investissement de l'AECG est compatible avec le droit de $1^{\prime} U E^{(2)}$. À ce jour, quatorze États membres sur 28 ont ratifié l'AECG, alors qu'un recours est toujours pendant devant la Cour constitutionnelle d'Allemagne concernant la compatibilité du chapitre sur l'investissement avec sa Constitution ${ }^{(3)}$. Paradoxalement, alors que l'AECG offre la réponse la plus étoffée aux préoccupations entourant l'arbitrage entre investisseur et État, il suscite lui-même la controverse la plus forte au sein de la société civile et dans plusieurs parlements des États membres de l'UE.

(1) CJUE, Slowakische Republik c Achmea, C-284/16, 6 mars 2018, ECLI:EU:C:2017:699.

(2) CJUE, Accord ECG UE-Canada, Avis 1/17, 30 avril 2019, ECLI:EU:C:2019:341.

(3) Voir Allemagne, Bundesverfassungsgericht, Communiqué $n^{\circ} 71 / 2016$, « Application for Preliminary Injunction in the "CETA" Proceedings Unsuccessful $\gg(13$ octobre 2016), en ligne: https://www.bundesverfassungsgericht.de/SharedDocs/Pressemitteilungen/ EN/2016/bvg16-071.html. 


\section{Troisième modèle : l'abandon de l'arbitrage entre investisseur et État dans l'ACEUM}

L'ACEUM a été signé le 30 novembre 2018 et n'est toujours pas entré en vigueur. Un protocole d'amendement signé le 10 décembre 2019 devrait faciliter la finalisation de la ratification de l'accord, sans qu'il ne touche aux dispositions d'origine sur l'investissement ${ }^{(1)}$. L'accord est le fruit de la renégociation de l'Accord de libre-échange nord-américain ${ }^{(2)}$ (ALENA) demandée par les États-Unis. L'investissement est un sujet qui connait d'importants changements avec l'ACEUM. Le chapitre 11 sur l'investissement est assurément l'un des plus connus de l'actuel ALENA, en raison des importantes controverses qu'il a vu naître du fait des contestations de mesures environnementales par des entreprises privées contre le Canada, mais aussi contre les ÉtatsUnis et le Mexique. Ces controverses ne sont pas étrangères aux modifications significatives qui lui sont apportées - à la demande du gouvernement américain - dans le nouveau chapitre 14 sur l'investissement de l'ACEUM. Les règles matérielles prescrivant

(1) Protocole d'amendement de l'Accord entre le Canada, les États-Unis et le Mexique, 10 décembre 2019, en ligne : https://www.international.gc.ca/tradecommerce/assets/pdfs/agreements-accords/cusma-aceum/protocolamendment_protocole-amendement_fra.pdf [Protocole de 2019].

(2) Accord de libre-échange nord-américain entre le gouvernement du Canada, le gouvernement des États-Unis et le gouvernement du Mexique, 17 décembre 1992, RT Can $1994 n^{\circ} 2$. 
les normes de protection des investissements étrangers sont certes reconduites. On retrouve en effet les mêmes clauses du traitement national ${ }^{(1)}$, de la $\mathrm{NPF}^{(2)}$, du TJE et de l'expropriation directe et indirecte $^{(3)}$, avec la clarification de cette dernière notion dans une annexe, ainsi que la réaffirmation du droit de légiférer de l'État ${ }^{(4)}$. En revanche, l'arbitrage entre investisseur et État qui est à l'origine des controverses passées est complètement abandonné par le Canada dans ses rapports avec les États-Unis et le Mexique ${ }^{(5)}$. Il s'agit a priori d'un gain pour le Canada, qui a été poursuivi 27 fois par des investisseurs américains depuis 1994. À noter qu'un régime transitoire de trois ans est prévu afin de maintenir temporairement l'accès à l'arbitrage pour les investissements réalisés avant l'entrée en vigueur de $\mathrm{l}^{\prime} A C E U M^{(6)}$. À la demande du gouvernement mexicain sortant, l'arbitrage entre investisseur et État a été maintenu entre les États-Unis et le Mexique, afin d'éviter l'annulation d'importantes réformes réglementaires effectuées dans ce pays dans certains secteurs-clés de l'économie, comme le

(1) ACEUM, supra note 3, art 14.4.

(2) Ibid art 14.5 .

(3) Ibid art 14.6, 14.8 et annexe 14-B.

(4) Ibid art 14.16 .

(5) Ibid annexe 14-D, art 14-D.1, sub verbo « Partie à l'annexe ».

${ }^{(6)}$ Ibid annexe 14-C, paragr 3. 
pétrole, l'électricité et les télécommunications ${ }^{(1)}$. En outre un recours à l'arbitrage plus limité est maintenu entre ces deux pays dans tous les autres secteurs de l'économie, mais seulement à l'égard de la violation de quelques règles matérielles du chapitre, dont surtout la clause d'expropriation limitée à l'expropriation directe $^{(2)}$. L'historique chargé du traitement des biens américains au Mexique au début du siècle dernier peut aussi expliquer ce maintien partiel de l'arbitrage.

L'abandon de l'arbitrage entre investisseur et État par le Canada constitue un changement important dans l'ACEUM dont il convient d'évaluer sommairement les conséquences. D'abord, il faut rappeler que l'arbitrage reste en vigueur dans les relations entre le Canada et le Mexique, puisqu'il est prévu par le chapitre sur l'investissement du PTPGP. Le véritable changement réside donc dans les relations entre le Canada et les États-Unis. L'abandon de l'arbitrage risque fort bien de ne pas signifier la fin des différends entre les investisseurs américains et le Canada, puisque le fait économique de l'investissement américain au Canada va continuer d'exister. La voie directe de l'arbitrage entre ces investisseurs et le Canada n'existant plus, c'est la manière dont ces différends se manifestent qui changera. Il est possible d'imaginer quatre

(1) Ibid annexe 14-E.

(2) Ibid annexe 14-D, art 14.D.1, sub verbo « Partie à l'annexe » et 14.D.3.1 
nouvelles voies pour la manifestation de ces différends. Premièrement, les entreprises américaines désireuses d'investir au Canada pourraient le faire indirectement par le truchement d'une société-écran ou d'une filiale établie dans un État tiers avec lequel le Canada a conclu un accord d'investissement prévoyant l'arbitrage entre investisseur et État. Elles bénéficieront ainsi de l'arbitrage prévu par ces accords, sous réserve des restructurations corporatives abusives ou d'une éventuelle clause de refus des avantages, qui permet précisément de bloquer les réclamations des sociétés-écrans ${ }^{(1)}$. Deuxièmement, comme le chapitre 14 de l'ACEUM prévoit toujours des règles de fond en matière d'investissement, les États-Unis pourraient eux-mêmes déposer une plainte contre le Canada pour violation de ces dispositions dans le cadre de son mécanisme interétatique de règlement des différends ${ }^{(2)}$, voire dans un autre forum international. Cette repolitisation du règlement des différends relatifs à l'investissement soulève toutefois une série de questions juridiques quant à son application, dont celle de savoir si les recours internes des investisseurs doivent être préalablement épuisés. Troisièmement, il demeurerait possible pour l'investisseur et le Canada de consentir à

(1) Voir généralement Tania Voon, Andrew Mitchell et James Munro, « Legal Responses to Corporate Manoeuvring in International Investment Arbitration » (2014) 5 Journal of International Dispute Settlement 41.

(2) ACEUM, supra note 3, art 31.1-31.22; Protocole de 2019, supra note 1, art 6. 
soumettre un différend à l'arbitrage moyennant un compromis spécifique à cette fin; la Nouvelle-Zélande adopte d'ailleurs explicitement cette approche dans le PTPGP avec certains États ${ }^{(1)}$. Enfin, les investisseurs américains pourraient aussi saisir les tribunaux canadiens de leur réclamation, mais le comportement $\mathrm{du}$ Canada se mesurera alors au droit canadien et non aux règles matérielles fixées par l'ACEUM. Ce contentieux mettra en lumière la portée de la protection offerte par le droit canadien ${ }^{(2)}$.

L'abandon pur et simple de l'arbitrage entre investisseur et État comme réponse aux préoccupations le concernant pourrait en dernière analyse être une solution plus néfaste que bénéfique si elle devait s'étendre. Le système est assurément perfectible, mais les problèmes à l'origine de la création de cette forme d'arbitrage ne manqueront pas de réapparaître s'il n'existe plus, à commencer par la politisation $\mathrm{du}$ règlement des différends relatifs à l'investissement et les risques de détérioration de la paix et de la sécurité internationales que cela peut entraîner.

(1) Voir l'analyse de cette nouvelle approche de la Nouvelle-Zélande au texte correspondant à la note 3 .

(2) Voir généralement Armand de Mestral, «Does Canadian Law Provide Remedies Equivalent to NAFTA Chapter 11 Arbitration? », dans Armand de Mestral, dir, Second Thoughts. Investor-State Arbitration between Developed Democracies, Waterloo (Ontario), Centre for International Governance Innovation, 2017, 155. 


\section{Conclusion}

À la lumière de ces développements récents dans la pratique conventionnelle des États et spécialement des pays industrialisés, quelle devrait-être la position de l'Égypte face à l'arbitrage entre investisseur et État? L'abandon pur et simple du système ou le retrait de la Convention de Washington ne semblent pas être des voies à privilégier pour un pays en développement qui souhaite continuer d'attirer des capitaux étrangers pour soutenir son économie. Les différends relatifs à de tels investissements ne disparaitront pas avec cette abolition ou ce retrait et ils ne seront que plus difficiles à régler. Il semble que l'Égypte devrait d'abord s'assurer de la cohérence entre ses propres accords en matière d'investissement. Elle devrait aussi privilégier l'amélioration du contenu matériel de ces accords ainsi que des règles sur l'arbitrage entre investisseur et État, en s'appuyant sur les travaux de la Conférence des Nations Unies pour le commerce et le développement (CNUCED) sur la réforme des accords internationaux sur l'investissement ${ }^{(1)}$. Enfin, l'Égypte devrait profiter de son appartenance à deux organisations régionales afin de s'engager activement dans le dialogue global sur la réforme de l'arbitrage entre investisseur et État et l'éventuelle création d'un

(1) Voir CNUCED, Investment Policy Framework for Sustainable Development, New York, Nations Unies, 2015, en ligne: CNUCED https://unctad.org/en/PublicationsLibrary/diaepcb2015d5 en.pdf. 
tribunal multilatéral de l'investissement. Il faut rappeler la création de la Cour arabe d'investissement dans le cadre de la Ligue des États arabes ${ }^{(1)}$, ainsi que l'adoption du projet de Code panafricain des investissements par l'Union africaine ${ }^{(2)}$. L'Égypte devrait aussi ne pas perdre de vue les besoins de protection des investisseurs égyptiens à l'étranger : les flux d'investissement Sud-Sud ou SudNord font en sorte qu'elle peut aussi se retrouver dans la position de l'État d'origine d'un investisseur connaissant des problèmes à

(1) Voir Convention unifiée pour l'investissement des capitaux arabes dans les États arabes, 26 novembre 1980, en ligne: Investment Policy Hub https://investmentpolicy.unctad.org/international-investment-

agreements/treaty-files/5616/download; Walid Ben Hamida, «The Arab League's Difficult Path Towards a Greater Quality and Clarity of Regional Investment Law : the Amended Arab Investment Agreement of 2013 Enters into Force » (2016) 8 International Journal of Arab Arbitration 5; Walid Ben Hamida, "The Development of the Arab Investment Court's Case Law : New Decisions Rendered by the Arab Investment Court» (2014) 6 International Journal of Arab Arbitration 3; Walid Ben Hamida, "The First Arab Investment Court Decision » (2006) 7 Journal of World Investment \& Trade 699.

(2) Union africaine, Commission de l'Union africaine, Département des affaires économiques, Projet. Code panafricain des investissements, Doc $\mathrm{n}^{\circ}$ EA15660-15 (2016), en ligne: Union africaine https://au.int/sites/default/files/documents/32844-doc-

projet code panafricain_dinvestissements decembre 2016.pdf; Makane Moïse Mbengue et Stefanie Schacherer, «The "Africanization" of International Investment Law: The Pan-African Investment Code and the Reform of the International Investment Regime » (2017) 18 Journal of World Investment \& Trade 414. 
l'étranger, comme l'illustre la réclamation récente d'un investisseur égyptien au CIRDI contre le Canada dans l'affaire Global Telecom Holding c Canada ${ }^{(1)}$.

(1) Global Telecom Holding c Canada, «Requête d'arbitrage », Aff CIRDI n ${ }^{\circ}$ ARB/16/16 (TBI Canada-Égypte, 6 juin 2016). 